\title{
Tail regression in Ciona intestinalis (Prochordate) involves a Caspase- dependent apoptosis event associated with ERK activation
}

\author{
Jean-Philippe Chambon ${ }^{1}$, Jonathan Soule ${ }^{1}$, Pascal Pomies ${ }^{2}$, Philippe Fort ${ }^{3}$, Alain Sahuquet ${ }^{1}$, \\ Daniel Alexandre ${ }^{1}$, Paul-Henri Mangeat ${ }^{1}$ and Stephen Baghdiguian ${ }^{1, *}$ \\ ${ }^{1}$ UMR 5539 Centre National de la Recherche Scientifique, Dynamique Moléculaire des Interactions Membranaires, Université \\ Montpellier II place E. Bataillon 34095 Montpellier cedex 05, France \\ ${ }^{2}$ CNRS FRE 2376, Génétique Moléculaire et Biologie du Développement, Villejuif, France \\ ${ }^{3}$ Centre de Recherche en Biochimie Macromoléculaire, CNRS-UPR 1086, 1919 Route de Mende, 34293 Montpellier cedex 5, \\ France \\ *Author for correspondence (e-mail: stbaghdi@univ-montp2.fr) \\ Accepted 9 April 2002
}

\section{SUMMARY}

Two apoptotic events take place during embryonic development of Ciona intestinalis. The first concerns extraembryonic cells and precedes hatching. The second controls tail regression at metamorphosis, occurs through a polarized wave originating from tail extremity, and is caspase dependent. This was shown by: (1) in vivo incorporation of a fluorescent marker of caspase activation in different cell types of the tail; (2) detection of an activated form of caspase 3-like protein by western blotting; and (3) failure of $30 \%$ of larvae to undergo metamorphosis after treatment of fertilized eggs with a pan-caspase inhibitor. In addition, Ciona embryos express a single ERK protein, specifically phosphorylated at

\section{INTRODUCTION}

Programmed cell death is one of the central cellular processes involved in development, aging, disease or stress response in metazoans (Hengartner, 2000; Rich et al., 2000; Meier et al., 2000; Yuan and Yankner, 2000). Comparative analyses of the components of the apoptotic machinery have shown that many of the proteins that play a crucial role in this process were already present in Porifera (Krasko et al., 1999; Wiens et al., 1999; Wiens et al., 2000) and Cnidaria (Cikala et al., 1999). Indeed, from hydra to man, the activation of caspases, a family of aspartic acid-directed proteases, is a conserved feature of cells undergoing apoptosis (Cikala et al., 1999).

The ascidian Ciona intestinalis, an invertebrate member of the Chordate phylum (Cameron et al., 2000), presents a spectacular metamorphosis. The swimming planctonic larval stage (tadpole) exhibits a remarkable chordate body plan, characterized by a dorsal neural tube and a typical notochord. Metamorphosis into a sessile filter feeder invertebrate involves an extensive reorganization of the body plan and most notably the regression of the tail. Tail regression during metamorphosis also occurs in amphibians and involves massive apoptosis. This raises the question of whether this metamorphosis. ERK activation was shown to be located in cells of the tail. Addition of MEK inhibitor in the culture medium prevented ERK activation and metamorphosis. In silico analysis of Ciona genome pointed to 15 caspases with high homology with humans, and a single ERK gene with high homology to both mammalian ERK1 and ERK2. It is concluded that the sequence of events leading to metamorphosis includes ERK phosphorylation followed by caspase-dependent apoptosis and tail regression.

Movies available on-line

Key words: Ciona, Metamorphosis, Apoptosis, Caspase, ERK mechanism is specific to amphibian or whether it was already present in early chordates.

Various mechanisms have been proposed to explain tail regression in ascidians. The first involved the contractile properties of the epithelial layer, which would induce the first phases of tail shortening. The second suggested that notochord cells develop contractile properties that would trigger tail shortening. The third proposed that tail regression would be caused by striated tail muscle contraction (reviewed by Cloney, 1978). However, the actual fate of these tail cells has remained unknown. As developmental events are rapid and highly reproducible in the context of a laboratory, Ciona is used as a very pertinent developmental model organism (Corbo et al., 2001; Satoh, 2001). We show that Ciona provides an exquisite model to study developmentally regulated apoptosis in the context of an organism that is equipped with the similar caspase complexity of a higher vertebrate.

\section{MATERIALS AND METHODS}

Animal husbandry

Adult Ciona intestinalis were collected in the Thau laguna (Herault, 
France). Oocytes and sperm were obtained by dissection of gonoducts and cross fertilization was performed in plastic petri dishes. Embryos were cultured at $18^{\circ} \mathrm{C}$ in $0.2 \mu \mathrm{m}$ filtered sea water containing 100 $\mathrm{U} / \mathrm{ml}$ penicillin, and $100 \mu \mathrm{g} / \mathrm{ml}$ streptomycin.

\section{TUNEL staining and indirect immunofluorescence}

Tadpole larvae at the tail regression stage were fixed for 20 minutes with $3.7 \%$ formaldehyde in filtered seawater. Embryos were permeabilized with $0.2 \%$ Triton in phosphate-buffered saline (PBS) and washed once in PBS. The TUNEL staining was performed by two methods in order to visualize apoptotic cells in green or red fluorescence. The first TUNEL method (an in situ cell death fluorescein detection kit) was used according to the manufacturer's instructions (Boehringer). In the second TUNEL method, apoptotic cells were detected by incubating larvae for 1 hour at $37^{\circ} \mathrm{C}$ with biotin-16-dUTP (Roche Molecular Biochemicals) and terminal deoxynucleotidyl transferase (Promega). After the TUNEL reaction, embryos were washed three times in PBS and incubated for 45 minutes at room temperature with $\mathrm{Cy} 3$-conjugated streptavidin (Jackson ImmunoResearch Laboratories) diluted 1:500 in PBS. Larvae were then washed three times in PBS, once in TBS, rinsed in distilled water and mounted in mowiol. Positive controls were made by incubating larvae with $0.5 \mathrm{mg} / \mathrm{ml}$ DNAse 1 (Sigma) at room temperature for 10 minutes prior to TUNEL staining. DNAse 1treated sections incubated with fluorescein-labeled nucleotide mixture, without the addition of terminal deoxynucleotidyl transferase (TdT), were used as negative controls. The slides were analyzed with a Leica TCS 4D Laser Confocal Microscope. For double labeling studies, phosphorylated ERK was visualized with a polyclonal antibody raised in rabbit against the active form of MAP kinases ERK1 and ERK2 (di-phosphorylated 'HTGFLT(p)EY(p)VAT' peptide) (Cell Signaling Technology) and a Texas Red-conjugated goat-anti-rabbit immunoglobulin (Jackson Laboratories) as secondary antibody.

\section{Light and transmission electron microscopy (TEM)}

Larvae were fixed in $2.5 \%$ glutaraldehyde in $0.2 \mathrm{M}$ cacodylate buffer $(\mathrm{pH}=7.2)$, and post-fixed in $1 \%$ osmium tetroxide in $0.45 \mathrm{M}$ cacodylate buffer $(\mathrm{pH}=7.2)$. The fixed material was dehydrated in a graded alcohol series and embedded in Epon 812. For light microscopy semi-thin sections were stained with Toluidine Blue and observed on a Reichert microscope equipped with Nomarski optics. Images were captured from a 12 bits CCD camera (Princeton Instruments). For TEM, ultra-thin sections were classically contrasted with uranyl acetate and lead citrate, and observed with a Jeol 1200X transmission electron microscope.

\section{CaspACE in vivo incorporation}

The CaspACE FITC-VAD-FMK In situ Marker (Promega) was added at the hatching stage at a final concentration of $100 \mu \mathrm{M}$ in filtered seawater. Larvae were fixed and TUNEL stained as described above at the onset of tail regression [i.e. 24 hours post fertilization (hpf)].

\section{Treatments with caspase and MEK inhibitors}

The pan-caspase inhibitors (z-VAD-FMK and CaspACE FITC-VADFMK) were added at a final concentration of $100 \mu \mathrm{M}$ immediately after fertilization. Embryos were cultured at $18^{\circ} \mathrm{C}$ during 30 hours. Treated and control larvae were scored for signs of metamorphosis. The results were then statistically tested using variance analysis and were found to be significant at 0.05 . Results were the mean of four independent experiments for a total of 2012 untreated and 1881 treated larvae.

In two independent experiments, MEK inhibitor U0126 (Promega) was added at two final concentrations $(2$ and $6 \mu \mathrm{M})$ at $13 \mathrm{hpf}$ and embryos left to develop up to 30 hours. Results were scored from a total of 3493 (control), 2521 ( $2 \mu \mathrm{M} \mathrm{U0126)}$ and 2702 (6 $\mu \mathrm{M}$ U0126) larvae.

\section{SDS-PAGE and western immunoblotting}

Fertilized eggs were dechorionated with $1 \%$ sodium thioglycolate and $0.05 \%$ pronase $\mathrm{E}$ as described (Mita-Miyazawa et al., 1985) and thoroughly washed six times in $0.2 \mu \mathrm{m}$ filtered sea water. For caspase 3 analysis, larvae were sonicated on ice in RIPA lysis buffer $(150 \mathrm{mM}$ $\mathrm{NaCl}, 50 \mathrm{mM}$ Tris-Cl, pH 7.6, $5 \mathrm{mM}$ EDTA, 0.5\% NP-40, $1 \mathrm{mM}$ PMSF, $1 \mathrm{mM}$ orthovanadate, $0.5 \%$ sodium deoxycholate, $0.1 \%$ SDS) supplemented with Complete Protease Inhibitor Cocktail Tablets (Roche Molecular Biochemicals). Lysates were then clarified by centrifugation. Samples were diluted in sample buffer (Laemmli, 1970) and incubated at $85^{\circ} \mathrm{C}$ for 5 minutes. Total proteins were separated on $17.5 \%$ SDS gels and transferred onto PVDF membranes. The blots were blocked with 5\% milk powder in PBS-Tween, incubated with a polyclonal rabbit anti-caspase 3 (raised against the pro and activated forms, Santa Cruz) diluted 1:500 in PBS-Tween for 1 hour, washed in TBS-Tween and then incubated with the secondary antibody (HRP-conjugated anti-rabbit IgG antibody diluted 1:10000) for 1 hour and washed in PBS-Tween. Labeled proteins were detected using the ECL chemiluminescence kit (Amersham Pharmacia Biotech).

Additional analysis by western blotting with different antibodies were performed similarly using $12.5 \%$ SDS polyacrylamide gels containing $0.13 \%$ bisacrylamide. The various primary antibodies used were: rabbit anti-rat ERK1/2 antibody (Santa Cruz Biotechnology, Santa Cruz, CA), mouse monoclonal antibody against the active diphosphorylated form of MAP kinases ERK1 and ERK2 (raised against the di-phosphorylated 'HTGFLT(p)EY(p)VAT' peptide) (New England Biolabs), anti-rabbit skeletal myosin heavy chain and antichicken $\alpha$-tubulin (Sigma, Saint Louis, MO), anti-rabbit sarcomeric $\alpha$-actinin (ICN Biomedicals, Aurora, $\mathrm{OH}$ ), and anti-rat myogenin (BD Biosciences Pharmingen, San Diego, CA); the rabbit polyclonal directed against chicken $\alpha$-actinin-binding PDZ-LIM protein (ALP) was as described (Pomiès et al., 1999). Horseradish peroxidase linked to goat antibodies directed against rabbit immunoglobulins or mouse immunoglobulin Fc fragments (Sigma, Saint Louis, MO) were used as secondary antibodies.

\section{Cell culture}

The human Jurkat $\mathrm{T}$ cell line was cultured in RPMI 1640 medium supplemented with $10 \% \mathrm{FCS}, 100 \mathrm{U} / \mathrm{ml}$ penicillin and $100 \mu \mathrm{g} / \mathrm{ml}$ streptomycin. Cells were treated with $10 \%$ or $5 \%$ DMSO for 5 hours in order to induce apoptosis, then lysed with RIPA lysis buffer (150 $\mathrm{mM} \mathrm{NaCl}, 50 \mathrm{mM}$ Tris-Cl, pH 7.6, 5 mM EDTA, 0.5\% NP-40, $1 \mathrm{mM}$ PMSF, $1 \mathrm{mM}$ orthovanadate, $0.5 \%$ sodium deoxycholate, $0.1 \%$ SDS) supplemented with Complete Protease Inhibitor Cocktail Tablets (Roche Molecular Biochemicals).

The C2C12 myogenic cell line was grown as previously described (Pomiès et al., 1999).

\section{Acridine Orange incorporation and time-lapse movie}

Larvae at the early metamorphosis stage ( $24 \mathrm{hpf})$ were placed in a perfusion chamber continuously flowed with sea water containing 3aminobenzoic acid ethyl ester $(0.5 \mathrm{mM})$ and Acridine Orange (2 $\mu \mathrm{g} / \mathrm{ml})$. The pictures were collected every 5 minutes for 2 hours through the rhodamine channel of the Leica TCS 4D confocal microscope. The time lapse movie was made with Metamorph (Universal imaging).

\section{Database searches}

Searches in Ciona intestinalis genomic databases were done using TBLASTN and BLAST (Altschul et al., 1990) Department of Energy (DOE) web facilities. For all searches, the resulting sequences were deduced from at least three independent individual sequences.

\section{MAPK}

A single Ciona ERK2 protein sequence encoded by six exons was derived from the genomic sequences LQW190167.y1, 
LQW185341.x1, LQW78034.x1, LQW221663.y1, DEV44654.y1 and LQW243489.x1. p38 was deduced from the overlapping sequences LQW134919.y1, LQW208456.x1, LQW220041.y1, LQW259229.y2 and LQW42581.x1. Ciona ERK and p38 protein sequences were aligned with homologs from various species using ClustalW (Thompson et al., 1994).

$\alpha$-Tubulin

$\alpha$-tubulin exonic sequences were detected by TBLASTN. The complete gene was deduced from a contig reconstructed from the overlapping sequences LQW162562.y1, DEV14186.x1, LQW79054.y1, DEV7363.x1 and LWQ19150.y1.

\section{Caspases}

Ciona sequences homologous to the p20 domain of mammalian caspases were searched using TBLASTN. Fifteen independent Ciona caspase-like sequences were detected: CiCSP2a (LQW46573.x1), CiCSP2b (LQW202502.x1), CiCSP2c (LQW264446.x2), CiCSP2d (LQW190259.y1), CiCSP9 (LQW171787.x1), CiCSP3 (LQW237174.y1), $\quad$ CiCSP7c $\quad$ (LQW35987.y1), $\quad$ CiCSP7a (LQW160906.x1), CiCSP7b (DEV3140.y1), CiCSP6a (LQW237347.x2), CiCSP6b (LQW112173.x1), CiCSP6c (LWG743.y1), CiCSP6d (LQW134527.y01), CiCSP6e (DEV25998.y1) and CiCSP2f (LQW269851.x1).

\section{RESULTS}

\section{An apoptotic event is responsible for tail regression}

The swimming tadpole tail is composed of mononucleate striated muscle cells, arranged in three rows along each side of the notochord (Fig. 1A,B), which runs all along the tail and also extends into the caudal most part of the trunk. The notochord is covered dorsally by the neural tube, ventrally by the endodermal strand, and is surrounded by the tail muscles and the epidermis (Fig. 1B). The tunic is the most external structure of the tail. The nucleus of muscle cells is centrally located, with the surrounding cytoplasm packed with mitochondria (Fig. 1C). Each muscle cell contains approximately 30 myofibrils (Fig. 1D), which are all aligned in parallel, slightly oblique to the long axis of the cell (Fig. 1C). At this stage (18-24 hpf), no sign of apoptosis was detectable by TUNEL labeling (Fig. 1A), and this observation was confirmed by TEM (Fig. 1B,C) and the lack of in vivo incorporation of FITC-VAD-FMK caspase inhibitor (not shown).

A contrasted situation was observed during metamorphosis (24-30 hpf): Ciona tail cells died through apoptosis as first evidenced by TUNEL labeling (Fig. 2). Apoptosis originated at the tail extremity and evolved towards the base (Fig. 2A-C). This apoptotic wave was captured by time-lapse recording of Acridine Orange in vivo incorporation (see Movie). Except for neural cells, apoptosis was found to affect all other cell types at this developmental stage (Fig. 3), including striated muscle cells (Fig. 3B,F), notochord (Fig. 3G), highly vacuolated tunic cells (Fig. 3H) and epidermal cells (Fig. 3I). At the electron microscopy level, condensation of cytoplasm and nucleus (Fig. $3 \mathrm{G}$ ), separation of dying cells from their neighbors (Fig. 3B,C), and fragmentation into membrane-bound bodies (Fig. 3D,F,I) were observed. In spite of these cytological changes, organelles often remained intact and identifiable (Fig. 3E). All these features are hallmarks of apoptosis (Kerr et al., 1973).

Interestingly, the early stages of Ciona embryonic development (10-12 hpf) did not involve apoptosis. Indeed, no cell-death was detected by TUNEL in Ciona intestinalis embryos from the mid-tailbud-stage to metamorphosis (Fig. 4A,B). These results were confirmed by TEM (not shown). By contrast, maternal extra-embryonic cells underwent early programmed cell death, from the fertilized egg stage up to the mid-tailbud embryo. Apoptosis events were detected in both external (not shown) and internal follicle cells (Fig. 4A,B). These latter cells accumulated between the head and the tail of the future tadpole (Fig. 4B, arrow). Chromatin condensation in internal follicle cells was also observed by TEM. Upon
Fig. 1. Swimming tadpole does not shown features of apoptosis. (A) Swimming tadpole of Ciona intestinalis was labeled with TUNEL. Digitized images were merged to superimpose the TUNEL labeling (green pseudocolor field) over the respective Nomarski field. No sign of apoptosis was observed at this stage (Ot, otolith; Oc, ocellus; N, notochord). Double arrowed line indicates the level of the ultra-thin section shown in B. (B) Electron micrograph of cross-section of a Ciona tadpole tail at 20 hours post fertilization (hpf) (T, tunic; EP, epidermis; SM, striated muscle cell; NT, neural tube; ES, endodermal strand; NC, notochord). (C) Cross-section of a striated muscle cell from the tail of a Ciona intestinalis tadpole. The myofibrils (My) seen in the cross section are arranged in a single peripheral layer. The nucleus $(\mathrm{N})$ and mitochondria $(\mathrm{m})$ are present in the internal core. (D) Striated muscle cell tangentially sectioned and showing one sarcomere. Scale bars: $380 \mu \mathrm{m}$ in $\mathrm{A} ; 7 \mu \mathrm{m}$ in $\mathrm{B} ; 3 \mu \mathrm{m}$ in $\mathrm{C}$; $0.4 \mu \mathrm{m}$ in $\mathrm{D}$.

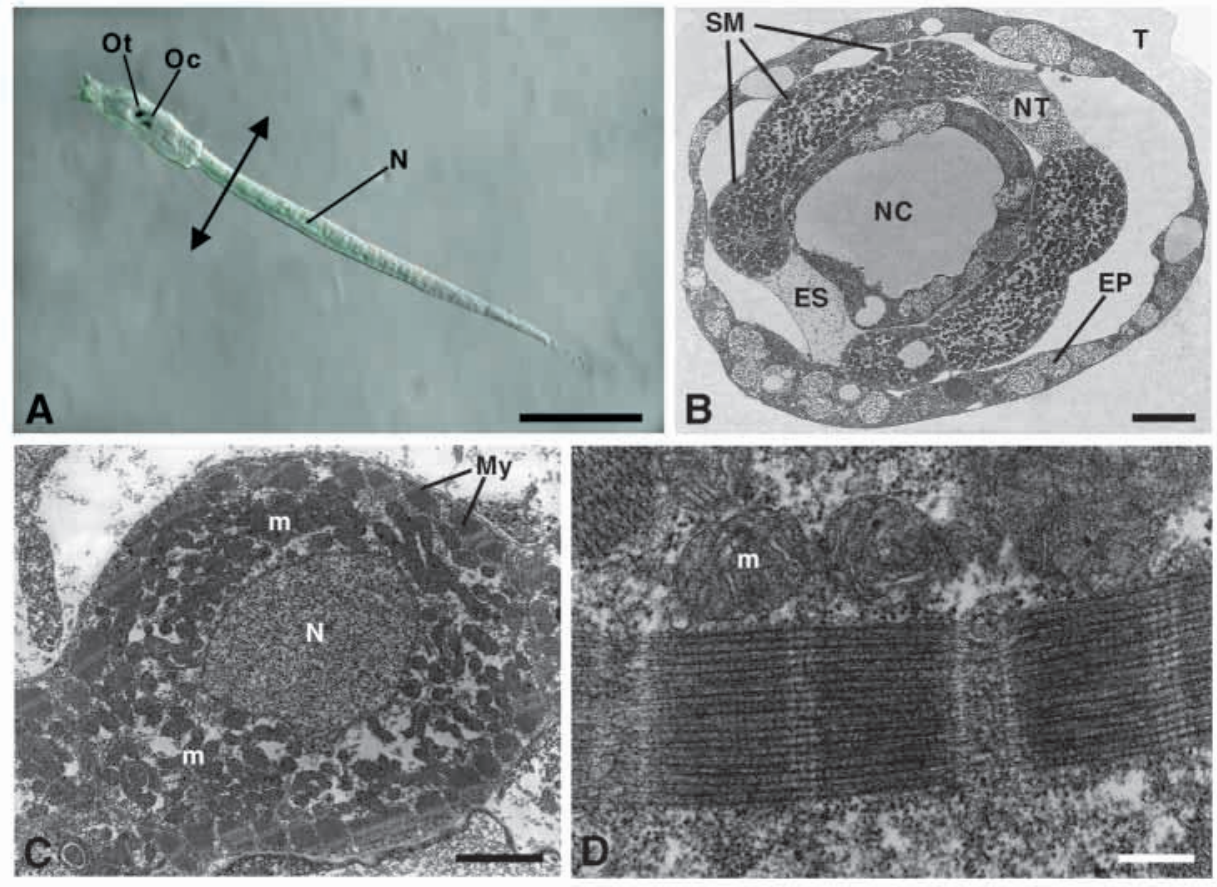



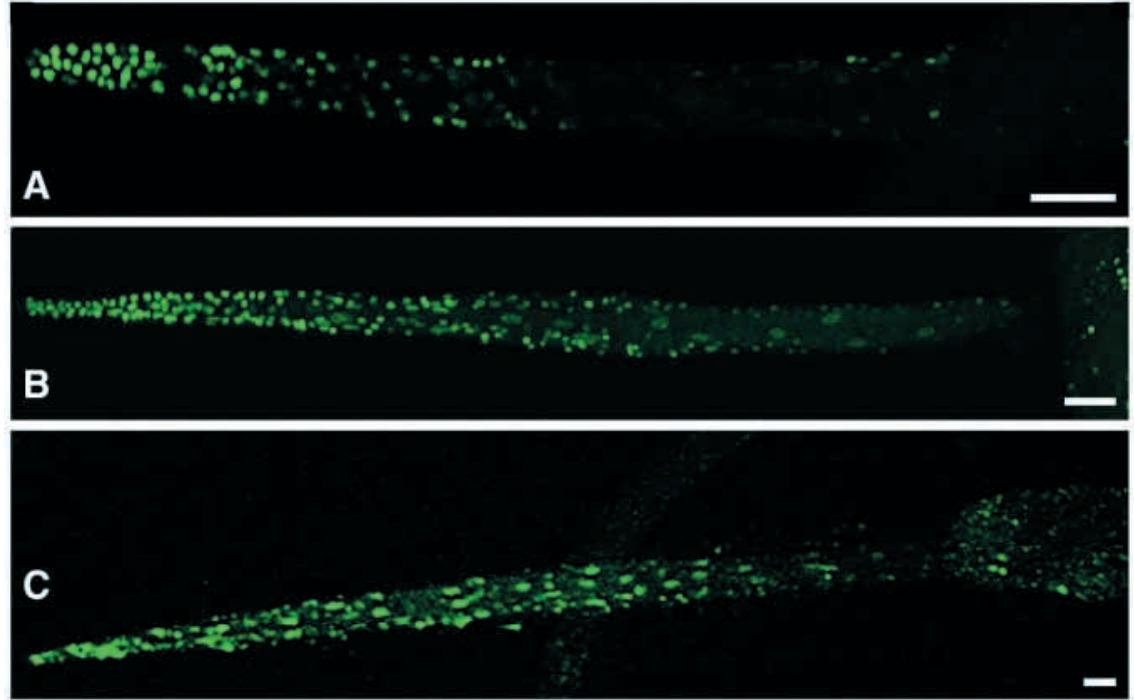

Fig. 2. TUNEL labeling of Ciona intestinalis tadpole at successive stages (A-C) of the tail regression. Apoptotic nuclei appear in green. Scale bars: $220 \mu \mathrm{m}$ in A; $140 \mu \mathrm{m}$ in B; $80 \mu \mathrm{m}$ in $\mathrm{C}$. progression of apoptosis, the nuclear membrane disappeared and the chromatin condensed characteristically (Fig. 4C,D), while a new endo-membrane system, probably derived from the endoplasmic reticulum, enclosed cytosolic regions (Fig. 4D, arrow).

\section{A caspase 3-like protein is induced and activated at the apoptosis/tail regression stage}

We next examined whether apoptosis was caspase dependent. We first searched Ciona genomic sequences for the presence of caspase p20/p10 domains (Cerretti et al., 1992; Thornberry et al., 1992). Fifteen independent sequences showed significant homology $\left(P<10^{-6}\right)$, most of which containing the ICE active site QACRG (Fig. 5). Although all the complete protein sequences remain to be accurately determined, this indicates that Ciona may potentially express as many caspases as vertebrates do.
Fig. 3. Apoptosis of striated muscle, notochord, epidermis and tunic cells 26 hours postfertilization. (A) Superposition of TUNEL and wheat-germ agglutinin (WGA) labeling (apoptotic nuclei appear in green, tadpole in red). Double arrowed line indicates the level of the semi-thin section shown in B. (B) Transverse section performed in the tail observed using Nomarski differentialinterference optics. One of the six muscle cells was clearly replaced by apoptotic body (ab) (T, tunic; TC, tunic cell; SM, striated muscle cells; NC, notochord). (C) Higher magnification of the apoptotic body shown in the rectangle in $\mathrm{B}$ (EP, epidermis). (D,E) Extracellular muscle apoptotic bodies, one with large vacuoles (lv) and a nuclear fragment (nf) containing condensed chromatin (cc). (E) At later stage of apoptosis, nuclear membrane disappears around the condensed chromatin. (F) Striated muscle cell at earlier stage of apoptosis. Note the presence of large vacuoles (lv) around the nucleus, the chromatin condensation (arrow) and the well preserved mitochondria (m). (G) NC showing chromatin condensation (arrow). (H) Highly vacuolated TC with condensed nucleus (arrow). Note the importance of the endoplasmic reticulum (er) in cytoplasm of epidermal cells (EP). (I) Typical feature of apoptotis in epidermal cell. Nuclear fragments (nf) containing condensed chromatin (cc) are wrapped by multiple membranes $(\mathrm{mm})$. The membranes seem to be derived from endoplasmic reticulum as suggested by the presence of ribosome-like particle (rp, arrowhead). Some vacant spaces (v) appear to grow between the wrapped fragment resulting in their separation. Scale bars: $180 \mu \mathrm{m}$ in A; $10 \mu \mathrm{m}$ in $\mathrm{B} ; 4 \mu \mathrm{m}$ in $\mathrm{C} ; 1.7 \mu \mathrm{m}$ in $\mathrm{D} ; 1.6 \mu \mathrm{m}$ in $\mathrm{E} ; 2.7 \mu \mathrm{m}$ in $\mathrm{F} ; 3 \mu \mathrm{m}$ in $\mathrm{G}, \mathrm{H} ; 1.2 \mu \mathrm{m}$ in $\mathrm{I}$.
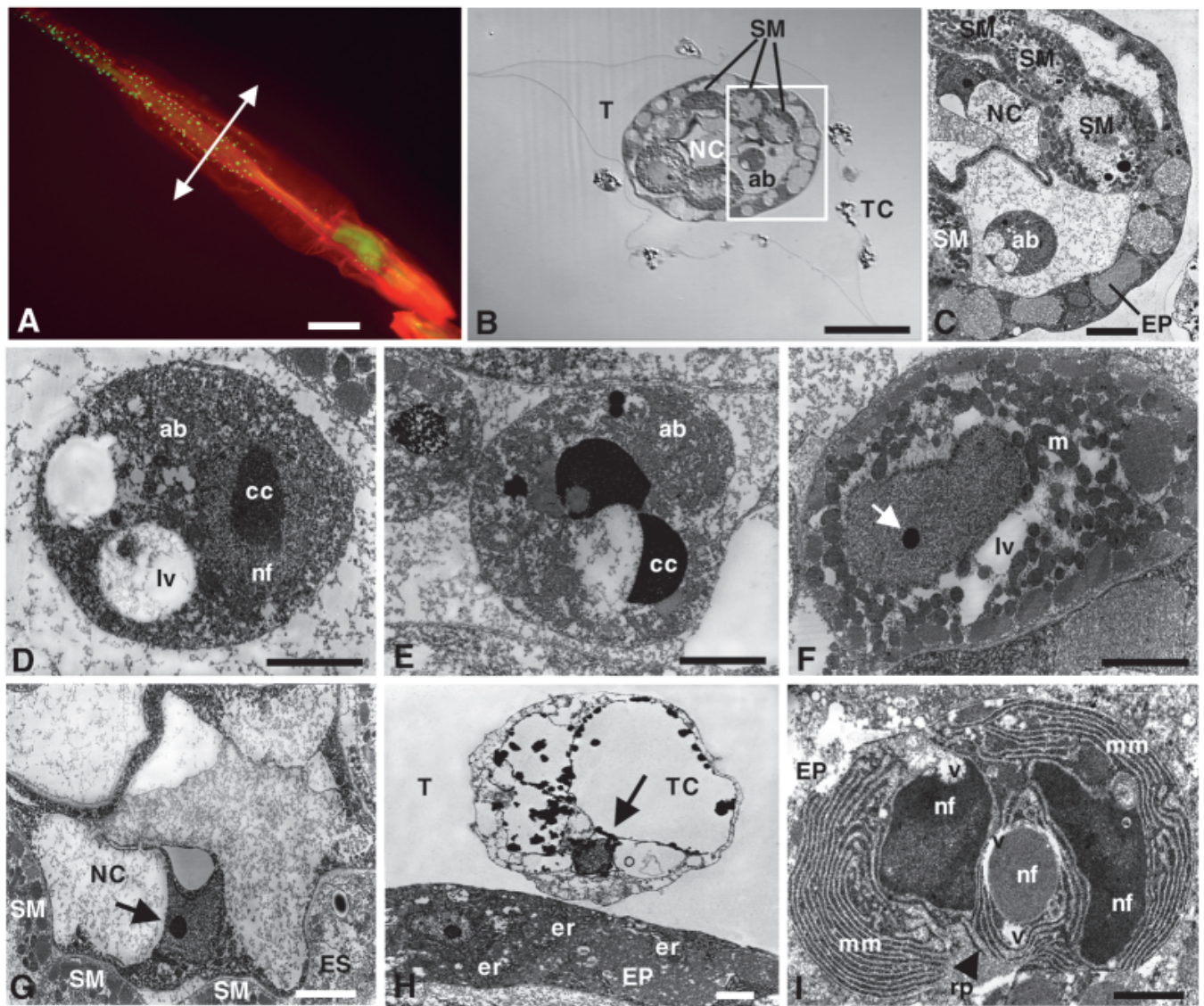
Given the high number of potential caspases and their similarity with human caspases, we next sought to identify caspase-like proteins from Ciona extracts using an anti-caspase antibody directed against human caspase 3. Ciona larvae at the mid-tailbud (10-12 hpf), swimming (18-24 hpf) and metamorphosis (28 hpf) stages were analyzed by western blotting (Fig. 6). The level of total protein of each sample was visualized by Coomassie Blue staining (Fig. 6; upper panel). Cell extracts from Jurkat cells treated (lanes 3, 9, 10) or not (lanes 6) with DMSO (an inducer of apoptosis) helped as a positive control for caspase 3 detection and activation. A caspase-like protein was found to be present only in the pool of metamorphosing larvae (lane 8). Interestingly, two bands at a molecular weight corresponding to a pro- and activated form of caspases were detected at this stage, when compared with the Jurkat positive controls. This caspase-like protein was absent in mid-tailbud embryos and swimming larvae (lanes 1, $2,4,5,7)$. All results strongly suggests that at tail regression stage, a caspase-like protein is expressed and activated to trigger apoptosis.

In a next step, a fluorescent marker of activated caspases (FITC-VAD-FMK/caspACE), was added to the incubation medium of swimming tadpoles prior to apoptosis (18 hpf). During the subsequent metamorphosis and tail regression, approximately $50 \%$ of TUNEL-positive cells were also found caspACE positive (Fig. 7A-C), thereby confirming that caspase-like proteins were activated in vivo during tail apoptosis. This caspase-like activation was obvious in striated muscle cells (Fig. 7D), suggesting that the striated muscle apoptosis observed by TEM (Fig. 3B-F) is a caspase-dependent phenomenon.

Finally, we studied the effect of treatment of larvae by pancaspase inhibitors. Fertilized eggs were incubated at $18^{\circ} \mathrm{C}$ in
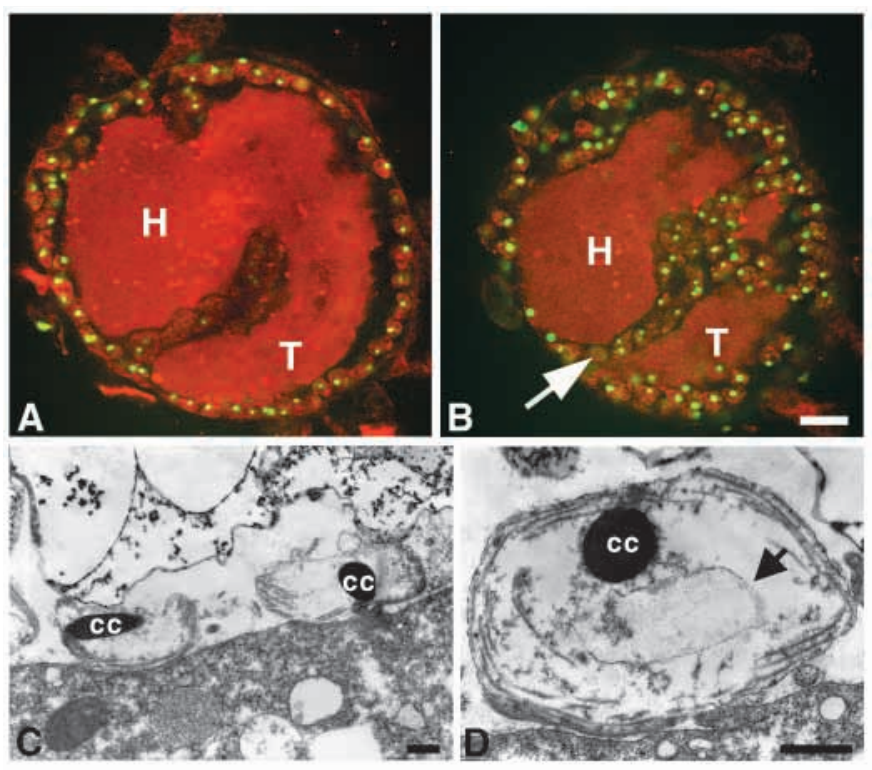

Fig. 4. Extra-embryonic apoptosis in internal follicle cells at midtailbud stage (12 hpf). (A) Confocal optical section showing the superposition of TUNEL and WGA labeling in a mid-tailbud embryo. Apoptotic nuclei of internal follicle cell appear in green, cytoplasm in red. No apoptosis is seen in the head $(\mathrm{H})$ or in the tail (T) of the future tadpole. (B) Optical section at a different focal plane of the embryo shown in (A). Note the accumulation of apoptotic internal follicle cells between the head and the tail (arrow). (C,D) Apoptotic internal follicle cells observed by TEM at midtailbud stage (12 hpf). The nuclear membrane disappears when apoptosis progresses to result in a rounded condensed chromatin (cc), while a new membrane system encloses narrow regions of the cytosol (arrow). Scale bars: $85 \mu \mathrm{m}$ in A,B; $1.4 \mu \mathrm{m}$ in C; $1.2 \mu \mathrm{m}$ in D.
Fig. 5. Partial sequence alignment of Ciona and human caspases. Ciona sequences were aligned and clustered using ClustalW (Thompson et al., 1994) with human sequences: HsCasp2, XP_036975 (amino acids 243301); HsCasp9, XP_048851 (amino acids 220-285); HsCasp10, NP_001221 (amino acids 298356); HsCasp8, XP_054990 (amino acids 285-343); HsCasp3, XP_054686 (amino acids 104161); HsCasp7, NP_203125 (amino acids 127-184); HsCasp6, XP_003600 (amino acids 15-72). Three main clusters (framed) were obtained: CiCSP2a, b, c and d, related to human caspase 2 ; CiCSP9, related to human capsases 8, 9 and 10; and a larger cluster including three subgroups CiCSP3 and human caspases 3 and 7, CiCSP7a-c and CiCSP6a-e. These latter are related to human caspase 6 . For each cluster, residues shared by at least $80 \%$ and $60 \%$ sequences are indicated in red and blue, respectively. A consensus sequence is indicated below each frame. Conserved residues in each cluster or subgroup are shaded. Residues corresponding to the ICE pentapeptide are underlined.

\begin{tabular}{|c|c|}
\hline HsCasp2 & - GAIYGVDGKLLQLOEVFQLFDNANCP SLONKPKMFFIQACRG \\
\hline Cic\$p2a & --TDIYASDGRSIPIESLIKMFDNVNCPRLRNKEKIFFIQCCRG \\
\hline Cicsp2b & ELEEHYYNSCSLVAAMSHGDA------GCFYGSDGVSVAIDTVVNFF SNQNCHSLQRKPKIFLFQACQG \\
\hline Cicsp2c & $---G K I I T E D G V-V S I L D F M E S F V S S R C P A I M G K P K I F L F F$ \\
\hline Cicsp2d & DNELNNRFCLWVUYVLAHGTKG-----QQIYGVDGKCUSVREHTMKPF LHNCSOITNKPKLFFVDACOG \\
\hline Consensus & 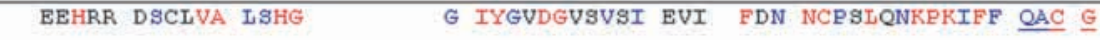 \\
\hline HsCasp9 & QFPGAVYGTDGCPVSVEKIVNIFNGT SCP SLGGKPKLFFIQACGG \\
\hline Cicsp9 & -GCUYGVDGLSLSTSKISKSFRPGMCSSLTGKPKIPFFOACQG \\
\hline HsCasp10 & -- GAVYS\$DEALIPIREIMSHFTA-QCPRLAEKPKLFFIQACQG \\
\hline HзСазр8 & QLMDHSMMD CFICCILSHGDK-------GIIYGTDGQEAPIYELTSQFTG-KCPSLAGKPKVFFIOACQG \\
\hline Consensus & I I S FTG CPSLAGKPKLFFIQACQG \\
\hline HsCasp3 & -GIIFGTNGPUDI-KKITNFFRG-RCRSITGKPKLFIIIQACRG \\
\hline CiCsp3 & -GVLYTKDGDIHL-KEILDSFKA-RCPSLAGKEKLFFIOACRG \\
\hline HsCasp 7 & --NVIYGKDGUTPI-KDLTAHFRG-RCKTLLEKPKLFFICACRG \\
\hline Cicsp7e & ---DVLYARDDKMEL-KDFMQPFRG-NCPSLATKPKLFFIQ \\
\hline Cicsp7a & -NILYSHDDEMKL-KDFTTPFEADKCRSLASKEKLFFVOACRG \\
\hline Cicsp $7 \mathrm{~b}$ & -EMVYAKDDSMKL-KTLISRVSA-ECPSLAGKPKLFFVQACRG \\
\hline HsСasp6 & STVSHADADCFVCVFLSHGEG----NHIYAYDAKIEI-QTLTGLFKGDKCHSLVGKPKIFIIQACRG \\
\hline cicsp6a & 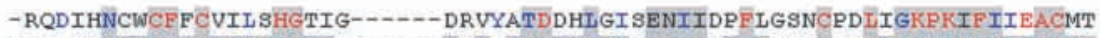 \\
\hline cicsp 6b & --GVIYTTDGKLDIOENILNKFMPODC--LKGKPKIFIFOACRG \\
\hline Cic\$p $6 \mathrm{f}$ & IGHGRV-----GFIKTTDGKIDIQDDILDKFMSHKC--LEKKPKLFIFQACRG \\
\hline cicsp6c & --- NPLI ICFICMFIGHGGP------GFINTAD GRLDIHENIL SRFMPQDC--LKGKRKIFIFQACSG \\
\hline Cic\$p 6d & KLKSENNYSCFICMFIGHRSD------GCIKATDGKIDIHENILAKFMTHKC--LEGKEKIFIFQACRG \\
\hline Cicspbe & ADGVOEEDQCFLGMFIGHGSP------GTVESRHGSLGIHSKIIPMFARIEW--LKGKPKIFVFOACRG \\
\hline Eor & G IYATDGKLDIHK II F G CPSL GKPKLFIIQACRG \\
\hline
\end{tabular}


Fig. 6. Caspase 3 like protein is expressed and activated during Ciona intestinalis metamorphosis. Comparative western blot analysis of Ciona tissue homogenates, with antibody against human caspase 3 . Coomassie Blue-stained gel (CB) showing the total proteins from Ciona (top). Western blotting (WB) was performed at various stages of embryonic development and metamorphosis (bottom).

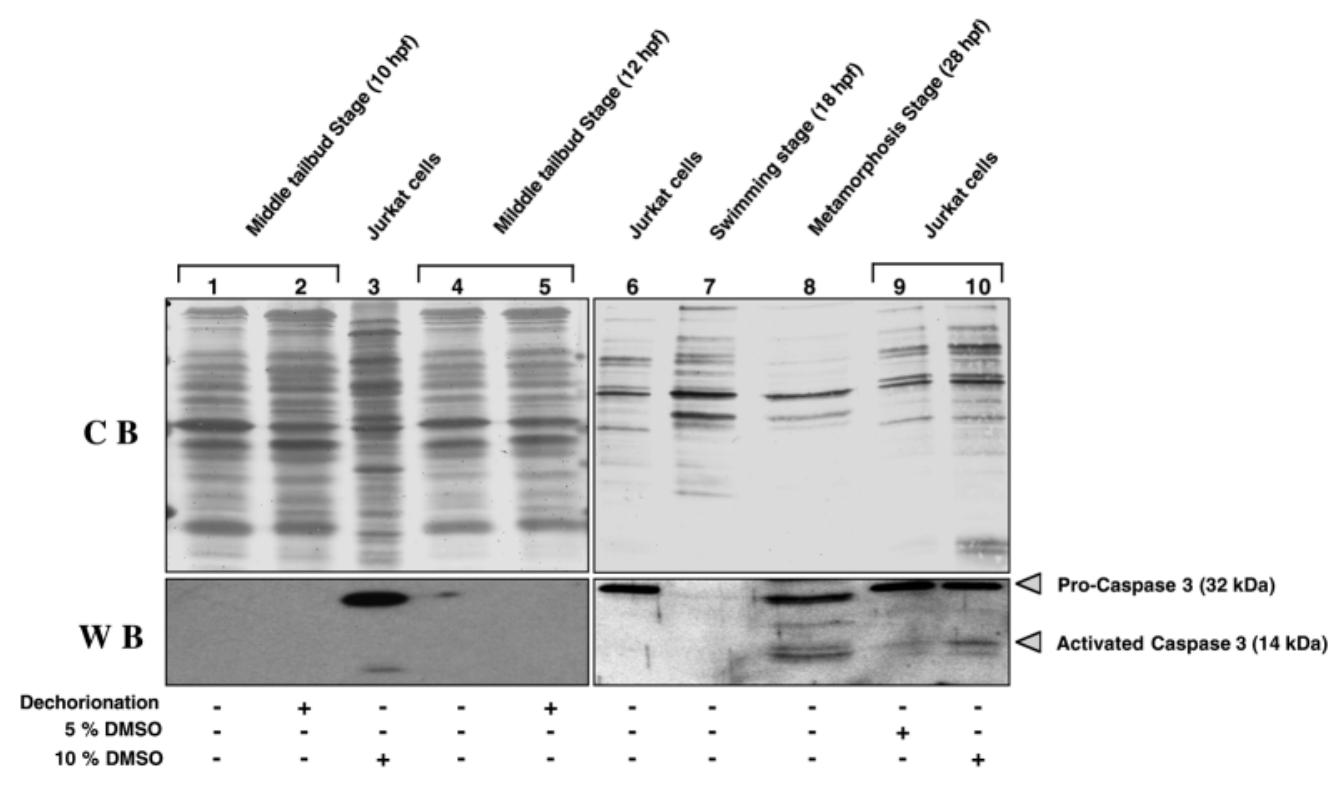

chain, $\alpha$-actinin-binding PDZ-LIM protein (ALP), myogenin (not shown), $\alpha$-tubulin and ERK1/2 (Fig. 8). Only the two latter proteins were positively detected at an expected molecular weight. A highly conserved $\alpha$-tubulin sequence was

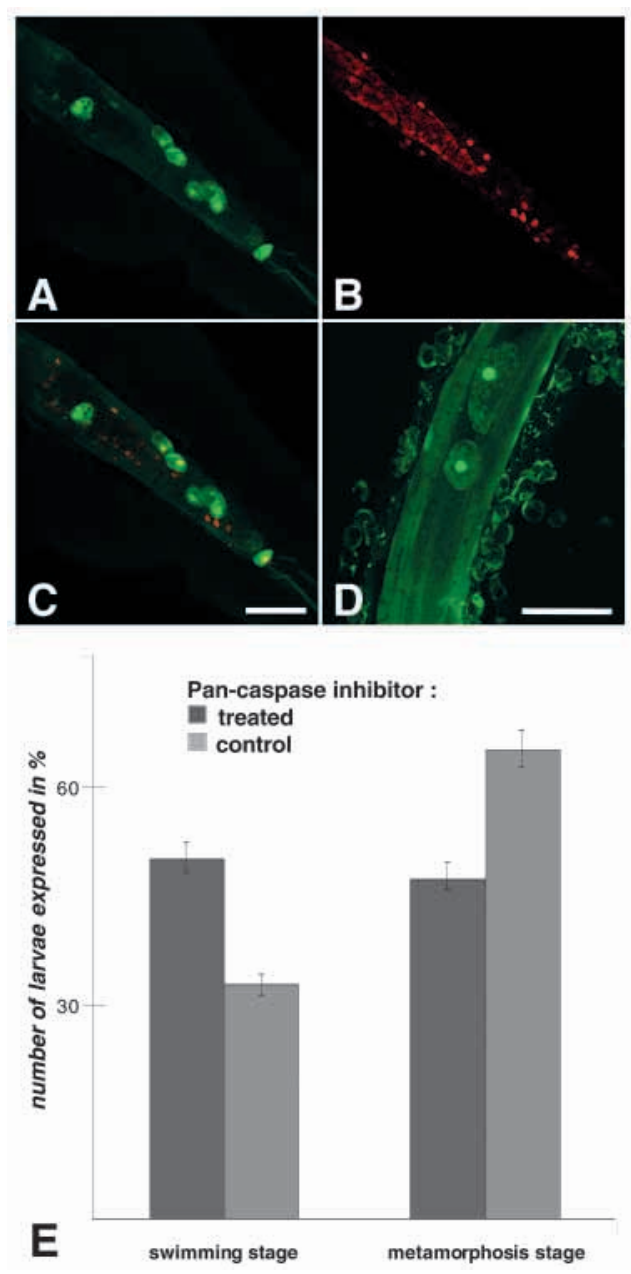

Fig. 7. Direct detection of caspase activity in situ using the viable reagent CaspACE ${ }^{\mathrm{TM}}$ FITC-VAD-FMK. (A-C) Double-detection of activated caspase and apoptosis in the tail of Ciona at metamorphosis stage (28 hpf). TUNEL labeling was performed after in vivo incorporation of caspACE/FITC-VAD-FMK. Digitized images were merged to superimpose the caspACE-labeled cells (green pseudocolor field) over the respective TUNEL-labeled field (TUNELpositive nuclei appear in red pseudo-color). Note the presence of caspACE-positive/TUNEL-positive cells (yellow nuclei/green cytoplasm) that co-exist with caspACE-positive/TUNEL-negative cells (green nuclei/green cytoplasm) and CaspACEnegative/TUNEL-positive cells (red nuclei). (D) In vivo incorporation of caspACE in striated muscle cells of Ciona tail at 28 hpf. (E) Pan-caspase inhibitor treatment blocks the metamorphosis of Ciona intestinalis. Data represent the mean of four independent experiments expressed as a percentage. Vertical bars correspond to the interval of confidence for percentage at $5 \%$. The results were assessed by variance analysis and were found to be significant

\section{Tail regression is associated with tubulin down} We next examined whether the expression of other proteins was affected during tail regression, among which we chose a set of cytoskeletal and signaling proteins. A differential screening of Ciona protein extracts was performed using antibodies directed against sarcomeric $\alpha$-actinin, myosin heavy

\footnotetext{
$(P<0.05)$. Scale bars: $80 \mu \mathrm{m}$.
} 


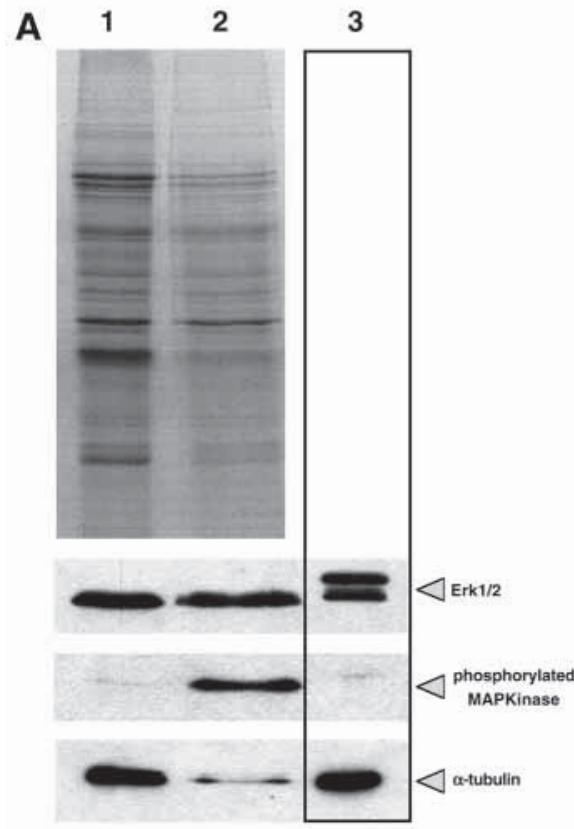

Fig. 8. (A) Detection of a MAP kinase isoform and tubulin in protein extracts from Ciona intestinalis. Coomassie Blue-stained gel showing the total proteins from Ciona intestinalis before (18 hpf) and after (24 hpf) induction of metamorphosis (upper panel; lanes 1 and 2, respectively). Corresponding western immunoblots probed with the anti-

ERK1/ERK2, anti-activated ERK1/ERK2 and anti-tubulin antibodies (lower panels). Western immunoblot analysis shows the presence of equivalent amount of a MAP kinase isoform in both Ciona intestinalis extracts, whereas activation of this MAP kinase is detected only after metamorphosis induction. $\alpha$-Tubulin breakdown is also observed at this stage. $\mathrm{C} 2$

C12 cells were used as control (lane 3). (B) Alignment of Ciona and human $\alpha$-tubulin. Ciona $\alpha$-tubulin (Ci $\alpha$-tub) was aligned with human $\alpha$ tubulin (Hs $\alpha$-tub, NP_006073). Conserved residues are indicated by a dash. Non-conserved residues are shaded. (C) Alignment of Ciona ERK and p38 with MAPK from other species. Ciona sequences were aligned with ERK2 from frog (X1ERK2, CAA42482.1), human (HsERK2, NP_002736.1), mouse (MmERK2, NP_036079.1) and fish (DrERK2, BAB11813.1), with ERK1 from human (HsERK1, AAH13992.1) and mouse (MmERK1, S28184), and with p38 from human (Hsp38, Q16539) and frog (Xlp38, P47812). Red indicates residues shared by all proteins; blue indicates residues shared by all ERKs; gray indicates consensus p38 residues; yellow indicates specific ERK1 residues; green indicates residues shared by ERK1 and CiERK2; the blue shading indicates epitope recognized by the ERK monoclonal anti-phosphotyrosine antibody.

also found in the genome database (Fig. 8B). Interestingly, $\alpha$ tubulin expression level was significantly reduced during metamorphosis (Fig. 8A, lane 2 lower panel), presumably as the result of the massive cell disorganization during the apoptosis-dependent tail regression stage.

With an antibody directed against human ERK1/2 proteins, a single $42 \mathrm{kDa}$ product was found equally expressed at both swimming (18 hpf) and early metamorphosis stages (24 hpf) (Fig. 8A). We thus searched Ciona genomic database and identified a unique putative protein termed $C i E R K$, showing 90 to $94 \%$ sequence similarity with mammalian ERK1 and ERK2 and whose $M_{\mathrm{r}}$ is fully compatible with the western blot signal (Fig. 8C). We next used a specific monoclonal antibody directed against the di-phosphorylated ERK1/2 'HTGFLT(p)EY(p)VAT' peptide (boxed in Fig. 8C). This peptide is present in $C i$ ERK but not in the Ciona form of p38 SAPK. Interestingly, upon metamorphosis $C i$ ERK was found to be specifically phosphorylated, arguing that an important step of CiERK activation takes place during metamorphosis. In order to locate which cells of the larvae were activated, we used a polyclonal antibody against the phosphorylated form of ERK, in combination with TUNEL staining (Fig. 9A-C). Indeed numerous nuclei of cells of the tail were found positively stained when $28 \mathrm{hpf}$ larvae were analyzed (Fig. 9C). In marked contrast, no activation was found at an earlier stage (18 hpf), when both apoptosis and metamorphosis were not yet induced (Fig. 9A,B). Interestingly, at $28 \mathrm{hpf}$, most of apoptotic nuclei were negative for ERK activation, suggesting that one molecular event, presumably ERK activation, preceded the other one, apoptosis, during the time sequence leading to tail regression. 
Fig. 9. Inactivation of Ciona ERK blocks metamorphosis. (A,B) Nuclei of cells of the tail are negative for ERK activation (red fluorescence) and apoptosis (green fluorescence) at $18 \mathrm{hpf}$ ( $\mathrm{A}$ is the phase contrast image of the same fluorescent field shown in B). (C) At $28 \mathrm{hpf}$, numerous nuclei of cells of the tail extremity are labeled either for ERK activation (red), apoptosis (green) or very rarely for both (yellow, arrow). Scale bars: $40 \mu \mathrm{m}$ in $\mathrm{A}, \mathrm{B} ; 25 \mu \mathrm{m}$ in $\mathrm{C}$.

(D) Extracts from untreated larvae at $18 \mathrm{hpf}$ (lane 1) and at $24 \mathrm{hpf}$ (lane 2), and larvae at $24 \mathrm{hpf}$ treated with $2 \mu \mathrm{M}$ U0126 MEK inhibitor (lane 3) were run on SDS-PAGE, stained with Coomassie brilliant blue (upper panel) and western blotted with the anti-phosphorylated ERK

monoclonal antibody (lower panel). (E) U0126 MEK inhibitor blocks the metamorphosis of Ciona intestinalis. Data represent the mean of two independent experiments expressed as a percentage of total number of larvae. Vertical bars correspond to the interval of confidence for

percentage at $5 \%$. The results were assessed by variance analysis and were found to be significant $(P<0.01)$. Note that during these experiments, a significant number of larvae did not hatch, but no effect of U0126 was detected at this stage.

Finally, we made use of an inhibitor of MEK, the kinase that phosphorylates and activates ERK, to assay to what extent ERK activation was involved in metamorphosis. Indeed, in larvae treated with $2 \mu \mathrm{M}$ U0126 MEK inhibitor, phosphorylation of ERK was very significantly decreased when compared with control untreated larvae (Fig. 9D). Moreover, the addition of two different concentrations of U0126 induced a $\sim 60 \%$ blockade of metamorphosis (Fig. 9E). These data demonstrated that ERK activation is a compulsory step for Ciona tail regression and metamorphosis.

\section{DISCUSSION}

Ciona metamorphosis entails a radical reconstruction of the body plan, from a swimming tadpole to a sessile filter feeder invertebrate. The massive destruction of obsolete tadpole tissues is an integral aspect of this transformation allowing for their replacement by adult tissues and structures. In this study, two different forms of apoptosis were recorded during the sequential events leading from hatching to metamorphosis of Ciona embryos and larvae. The first was linked to the caspaseindependent destruction of extra-embryonic maternal cells that takes place before, and possibly facilitates, hatching. The second consisted of a single massive caspase-dependent apoptosis wave that occurs at the metamorphosis stage and controls tail regression.

Whereas the ultrastructure of the swimming tadpole is well documented (reviewed by Satoh, 1994), little is known about the fate of Ciona intestinalis tail cells at metamorphosis stage. Using light and transmission electron microscopy, we showed unambiguously that during metamorphosis, these cells died by apoptosis. These included tunic, epidermal, notochord and striated muscle cells. This is in agreement with the degenerating figures observed during the metamorphosis of the solitary ascidian Herdmania momus (Degnan et al., 1996). Intriguingly, no sign of apoptosis was ever found in the neural tube. This observation is in agreement with a previous report showing a general absence of cell death in the nervous system of Ciona during embryonic development (Bollner and Meinertzhagen, 1993). The most striking result is the polarized origin of apoptosis that triggers cell regression. Through sequential TUNEL pictures, apoptosis started at the very tail extremity and was propagated through cell to cell up to the tail base. This was most obvious when in vivo Acridine Orange incorporation coupled to time-lapse videomicroscopy was viewed. This observation raises interesting questions: (1) what is the nature of the inductive signal that triggers apoptosis at the tip of the tail, and (2) what secondary signals transmit cell death from cell to cell. The microscopic data also document that no phagocytic event is linked to apoptosis as no cell cleaning process was observed. Instead, apoptotic cells accumulated in contact with the tunic before being shed into the surrounding medium.

One striking observation is that in general, metamorphosis is correlated with the definitive fixation of the larvae to the 
substratum. Upon adhesion, three types of larvae are successively observed: larvae with active tail, immobile larvae and metamorphosed larvae. It is tempting to correlate the induction of metamorphosis to the fixation step. However, in agreement with earlier reports (Just et al., 1981), a significant number of unfixed larvae were observed to undergo metamorphosis, bypassing the fixation step. If the general picture is that metamorphosis follows fixation, the correlation is not physiologically relevant. In other words, there were enough observed exceptions to rule out that the fixation step is the inductive trigger of metamorphosis. Our data favor the existence of a still ignored internal inductive mechanism that is independent of the fixation step. Recently, Patricolo et al. (Patricolo et al., 2001) demonstrated the presence of thyroxine in Ciona intestinalis larvae at the metamorphosis stage, suggesting that thyroxine might be one of the inductive signals.

Using complementary methods, we provided evidence that tadpole tail histolysis is a caspase-triggered programmed cell death response. By contrast, the molecular events controlling the early extra-embryonic cell death program appear to be caspase independent.

Using an antibody against human caspase 3, a specific immunological signal was detected in extracts from larvae that had undergone metamorphosis. The antibody was sensitive enough to detect a band of lower $M_{\mathrm{r}}$ compatible of what is expected for an activated form of caspase. That caspase signal was not detected at any other earlier stage, meaning that, upon metamorphosis, there is a specific expression of that particular protein.

In vivo incorporation of fluorescent caspACE confirmed that a caspase-dependent event occurred during tail regression. This inhibitor is known to associate with activated forms of caspases, and, indeed, a high proportion (50\%) of caspACE positive cells were also TUNEL positive. However, caspACEpositive and TUNEL-negative cells or caspACE-negative and TUNEL-positive cells were also detected during tail regression but at lower frequencies. CaspACE-positive, TUNEL-negative cells may be in the early stages of apoptosis; CaspACEnegative, TUNEL-positive cells may be in the final stage of apoptosis once caspase activation is terminated (Miho et al., 1999).

The addition in the medium, after egg fertilization, of the same fluorescent inhibitor or of a non fluorescent analog led to delayed metamorphosis as quantified 30 hours later by a significant increase in the number of swimming larvae and a reduction in the number of larvae that underwent metamorphosis when compared with untreated controls. These data added a definitive argument in the involvement of caspase(s) in polarized apoptosis and tail regression.

Whereas metamorphosis was blocked by addition of caspase inhibitor to the medium, it did not affect hatching (data not shown), a result in agreement with the recorded absence of caspase 3-like protein expression in the extracts from midtailbud stage embryos. Previous studies have supported a role for proteolytic enzymes leading to hatching of the tadpoles (Berrill, 1929; Caggegi et al., 1974; Denuce, 1975). We propose that these proteolytic enzymes are linked to, and presumably induced by, this apparent caspase-independent apoptotic event.

In silico genome anlysis led conclusively to the presence of 15 potential caspases, each with a high degree of homology to the vertebrates ones, a result with several implications. First, it adds a strong genetic support to the immunological data obtained with heterologous antibodies. Indeed, it validates the use of antibodies raised against human caspases, and gives additional confidence that the pro- and activated forms of Ciona caspase 3 have been correctly identified. Second, Ciona has the genomic capacity of controlling cell death with a comparable caspase equipment as higher vertebrates.

The use in Ciona of antibodies raised against proteins of higher vertebrates is of importance, because a very high number of them are immediately available. Especially interesting is the expression pattern obtained after analysis of extracts from pre- and post-metamorphosis larvae. Among a various set of antibodies that were tested, we identified two additional proteins that were significantly affected at metamorphosis.

The first protein that has been identified in our survey is Ciona tubulin. Tubulin protein expression was found to be strongly downregulated at metamorphosis. Tubulin turnover in cells is generally a slow event. Microtubule turnover in non-neuronal cells is, by comparison, very rapid. The downregulation of tubulin observed at metamorphosis presumably reflects the high level of cell disorganization that affects apoptotic cells.

Second, using an antibody raised against mammalian ERK1/2, a single band was identified in Ciona extracts and, more interestingly, the activated phosphorylated form of Ciona ERK was uniquely detected in the metamorphic extract, i.e. at a massive apoptosis stage. Additional in silico analysis provided a genomic confirmation of the existence of a single ERK gene made of a mixture of sequences found to be highly related to both human ERK1 and 2. The Ciona ERK gene is highly conserved, especially within the sequence from which the diphosphorylated peptide-directed antibody was generated. Therefore, Ciona ERK activation at metamorphosis, detected by this antibody, is fully compatible with in silico analysis. These results suggest that the phosphorylated form of ERK transduces the death-activating signal at the metamorphosis stage. Additional experimental data confirmed this conclusion, namely, the nuclear localization of activated ERK in cells of the tail and the effectiveness of MEK inhibitor U0126 to block ERK phosphorylation and metamorphosis. Double labeling with TUNEL staining indicated that TUNEL-positive nuclei very rarely colocalized with phosphorylated-ERK positive nuclei. This led to the suggestion that in normal larvae phosphorylation of ERK is a required step that precedes the onset of apoptosis.

The MAP kinase (ERK) pathway is usually involved in the suppression of apoptosis in mammalian somatic cells; however, ERK activation has been clearly linked to apoptosis in non-fertilized starfish eggs (Sasaki and Chiba, 2001). In this latter model, fertilization inactivates the MAP kinase (ERK) pathway and suppresses apoptosis. In addition, ERK has been reported both as an inducer of cytochrome c release with subsequent activation of the caspase pathway (Wang et al., 2000), as well as a controller of anoikis, the anchoragedependent form of apoptosis (Zugasti et al., 2001). In ascidians, EGF signaling in the anterior of the larva has been linked to settlement and potassium ions have been implicated in the development of an anterior signal center (Davidson and Swalla, 2001; Degnan et al., 1997; Eri et al., 1999). Therefore 
ERK activation in the tail could be a consequence of that signaling anterior pathway(s). If true, it will be interesting to understand how the signal is conveyed up to the tip of the tail where both ERK activation and apoptosis start.

We are particularly indebted to Michel Cantou and Laurent Libicz, the two divers of the 'Station Marine de Sète' (Université Montpellier 2) for harvesting Ciona. We also thank Drs J. L. Vayssière and B. Mignotte for the gift of the anti-caspase 3 antibody, Dr R. Hipskind for kind advice and the gift of antibodies directed against phosphorylated ERK. We also acknowledge Drs J. R. Bonami and P. Roch for the kind access to the local aquarium facilities, U. Hibner for helpful discussions, and S. Artero for statistical analysis.

\section{REFERENCES}

Altschul, S., Gish Miller, W., Myers, E. and Lipman, D. (1990). Basic local alignment search tool. J. Mol. Biol. 215, 403-410.

Berrill, N. J. (1929). Studies in tunicate development. I. General physiology of development of simple ascidians. Philos. Trans. R. Soc. Lond. Biol. 218, 37-78.

Bollner, T. and Meinertzhagen, I. A. (1993). The patterns of bromodeoxyuridine incorporation in the nervous-system of a larval ascidian, Ciona intestinalis. Biol. Bull. 184, 277-285.

Caggegi, S., Flugy, A., Puccia, E. and Reverberi, G. (1974). On the hatching of ascidian larvae. Rend. Accad. Naz. Lincei. Ser. 8, 803-807.

Cameron, C. B., Garey, J. R. and Swalla, B. J. (2000). Evolution of the chordate body plan: new insights from phylogenetic analyses of deuterostome phyla. Proc. Natl. Acad. Sci. USA 97, 4469-4474.

Cerretti, D. P., Kozlosky, C. J., Mosley, B., Nelson, N., van Ness, K., Greenstreet, T. A., March, C. J., Kronheim, S. R., Druck, T., Cannizzaro, L. A. et al. (1992). Molecular cloning of the interleukin-1 beta converting enzyme. Science 256, 97-100.

Cikala, M., Wilm, B., Hobmayer, E., Böttger, A. and David, C. N. (1999). Identification of caspases and apoptosis in the simple metazoan Hydra. Curr. Biol. 9, 959-962.

Cloney, R. A. (1978). Ascidian metamorphosis: review and analysis. In Settlement and Metamorphosis of Marine Larvae (ed. F. S. Chia and M. E. Rice), pp. 255-282. Amsterdam: Elsevier.

Corbo, J. C., di Gregorio, A. and Levine, M. (2001). The ascidian as a model in developmental and evolutionary biology. Cell 106, 535-538.

Davidson, B. and Swalla, B. J. (2001). Isolation of genes involved in ascidian metamorphosis: epidermal growth factor signaling and metamorphosis competence. Dev. Genes Evol. 211, 190-194.

Degnan, B. M., Rohde, P. R. and Lavin, M. F. (1996). Normal development and embryonic gene activity of the ascidian Herdmania momus. Mar. Freshwater Res. 47, 543-551.

Degnan, B. M., Souter, D., Degnan, S. M. and Long, S. C. (1997). Induction of metamorphosis with potassium ions requires development of competence and an anterior signaling center in the ascidian Herdmania momus. Dev. Genes Evol. 206, 370-376.

Denuce, J. M. (1975). Proteolytic activity in Ciona intestinalis, associated with hatching. Arb. Internat. Physiol. Biochim. 83, 958-959.

Eri, R., Arnold, J. M., Hinman, V. F., Green, K. M., Jones, M. K., Degnan, B. M. and Lavin, M. F. (1999). Hemps, a novel EGF-like protein, plays a central role in ascidian metamorphosis. Development 126, 5809-5818.

Hengartner, M. O. (2000). The biochemistry of apoptosis. Nature 407, 770776.

Just, J. J., Kraus-Just, J. and Check, D. A. (1981). Survey of chordate metamorphosis. In Metamorphosis. A problem in Developmental Biology, 2nd edn (ed. I. L. Gilbert, and E. Frieden), pp. 265-326. New York: Plenum. Kerr, J. F. R., Harmon, B. and Searle, J. (1973). An electron-microscope study of cell deletion in the anuran tadpole tail during spontaneous metamorphosis with special reference to apoptosis of striated muscle fibres. J. Cell Sci. 14, 571-585.

Krasko, A., Schröder, H. C., Perovic, S., Steffen, R., Kruse, M., Reichert, W., Müller, I. M. and Müller, W. E. G. (1999). Ethylene modulates gene expression in cells of the marine sponge Suberites domuncula and reduces the degree of apoptosis. J. Biol. Chem. 274, 31524-31530.

Laemmli, U. K. (1970). Cleavage of structural proteins during the assembly of the head of bacteriophage T4. Nature 227, 680-685.

Meier, P., Finch, A. and Evan, G. (2000). Apoptosis in development. Nature 407, 796-801.

Miho, Y., Kouroku, Y., Fujita, E., Mukasa, T., Urase, K., Kasahara, T., Isoai, A., Momoi, M. Y. and Momoi, T. (1999). bFGF inhibits the activation of caspase-3 and apoptosis of P19 embryonal carcinoma cells during neuronal differentiation. Cell. Death Differ. 6, 463-470.

Mita-Miyazawa, I., Ikegami, S. and Satho, N. (1985). Histospecific acetylcholinesterase development in the presumptive muscle cells isolated from 16-cell-stage ascidian embryos with respect to the number of DNA replications. J. Embryol. Exp. Morphol. 87, 1-17.

Patricolo, E., Cammarata, M. and D'Agati, P. (2001). Presence of thyroid hormones in ascidian larvae and their involvement in metamorphosis. J. Exp. Zool. 290, 426-430.

Pomiès, P., Macalma, T. and Beckerle, M. C. (1999). Purification and characterization of an $\alpha$-actinin-binding PDZ-LIM protein that is upregulated during muscle differentiation. J. Biol. Chem. 274, 2924229250.

Rich, T., Allen, R. L. and Wyllie, A. A. (2000). Defying death after DNA damage. Nature 407, 777-783.

Sasaki, K. and Chiba, K. (2001). Fertilization blocks apoptosis of starfish eggs by inactivation of the MAP kinase pathway. Dev. Biol. 23, 18-28.

Satoh, N. (1994). In Developmental Biology of Ascidians (ed. P. W. Barlow, D. Bray, P. B. Green and J. M. W. Slack), pp. 234. Cambridge: Cambridge University Press.

Satoh, N. (2001). Ascidian embryos as a model system to analyze expression and function of developmental genes. Differentiation 68, 1-12.

Thornberry, N. A., Bull, H. G., Calaycay, J. R., Chapman, K. T., Howard, A. D., Kostura, M. J., Miller, D. K., Molineaux, S. M., Weidner, J. R. and Aunins, J. (1992). A novel heterodimeric cysteine protease is required for interleukin-1 beta processing in monocytes. Nature 356, 768-774.

Thompson, J. D., Higgins, D. G. and Gibson, T. J. (1994). CLUSTAL W: improving the sensitivity of progressive multiple sequence alignment through sequence weighting, position-specific gap penalties and weight matrix choice. Nucleic Acids Res. 22, 4673-4680.

Wang, X., Martindale, J. L. and Holbrook, N. J. (2000). Requirement for ERK activation in cisplastin-induced apoptosis. J. Biol. Chem. 275, 3943539443.

Wiens, M., Khoziol, C., Hassanein, H. M. A., Müller, I. M. and Müller, W. E. G. (1999). A homolog of the putative tumor suppressor QM in the sponge Suberites domuncula: downregulation during the transition from immortal to mortal (apoptotic) cells. Tissue Cell 31, 163-169.

Wiens, M., Krasko, A., Müller, C. I. and Müller, W. E. G. (2000). Molecular evolution of apoptotic pathways: cloning of key domains from sponges (Bcl2 homology domains and death domains) and their phylogenetic relationships. J. Mol. Evol. 50, 520-531.

Yuan, J. and Yankner, B. A. (2000). Apoptosis in the nervous system. Nature 407, 802-809.

Zugasti, O., Rul, W., Roux, P., Peyssonnaux, C., Eychene, A., Franke, T. F., Fort, P. and Hibner, U. (2001). Raf-MEK-Erk cascade in anoikis is controlled by Rac1 and Cdc42 via Akt. Mol. Cell. Biol. 21, 6706-6717. 